

\title{
Atomic structure of grain boundaries in semiconductors
}

\author{
A. Bourret, J.J. Bacmann
}

\section{To cite this version:}

A. Bourret, J.J. Bacmann. Atomic structure of grain boundaries in semiconductors. Revue de Physique Appliquée, 1987, 22 (7), pp.563-568. 10.1051/rphysap:01987002207056300 . jpa-00245577

\section{HAL Id: jpa-00245577 https://hal.science/jpa-00245577}

Submitted on 1 Jan 1987

HAL is a multi-disciplinary open access archive for the deposit and dissemination of scientific research documents, whether they are published or not. The documents may come from teaching and research institutions in France or abroad, or from public or private research centers.
L'archive ouverte pluridisciplinaire HAL, est destinée au dépôt et à la diffusion de documents scientifiques de niveau recherche, publiés ou non, émanant des établissements d'enseignement et de recherche français ou étrangers, des laboratoires publics ou privés. 


\author{
Classification \\ Physics Abstracts \\ $61.70 \mathrm{~N}-61.16 \mathrm{D}$
}

\title{
Atomic structure of grain boundaries in semiconductors
}

\author{
A. Bourret and J. J. Bacmann $\left({ }^{+}\right)$ \\ IRF, DRF, Service de Physique, Groupe Structures, \\ $\left({ }^{+}\right)$IRDI, DMECN, Département de Métallurgie, SRM, CEN Grenoble, 85 X, 38041 Grenoble Cedex, \\ France
}

(Reçu le 10 octobre 1986, accepté le 12 janvier 1987)

\begin{abstract}
Résumé. - Cet article résume l'ensemble des observations en microscopie électronique (haute résolution, diffraction et franges- $\alpha$ ) sur les bicristaux de germanium. Les structures observées sont totalement isomorphes à celles qui existent dans le silicium. Elles tendent à former des arrangements bidimensionnels périodiques sur lesquels se superposent éventuellement des défauts linéaires. Sur les joints de flexion 〈011〉 il a été montré que l'environnement des atomes reste toujours tetraédrique par reconstruction des liaisons pendantes. L'activité électrique éventuelle de ces joints ne peut donc venir que de défauts ponctuels (intrinsèques ou impuretés).
\end{abstract}

\begin{abstract}
This paper summarizes the electron microscope observations (high resolution, diffraction and $\alpha$ fringes) on germanium bicrystals. Observed structures were found isomorphous to those of silicon. They tend to form a 2D-periodic medium with eventually additional linear defects. In pure tilt $\langle 011\rangle$ grain boundaries the tetrahedral coordination is always restored by dangling bond reconstruction. The electrical activity of such grain boundaries can therefore only be due to point defects (intrinsic defects or impurities).
\end{abstract}

\section{Introduction.}

The structure of special coincidence twins in semiconductors has been for several years subject to experimental and theoretical studies [1-4]. In particular a combination of high resolution electron microscopy (HREM) with electron diffraction and $\alpha$ fringes method on $\langle 011\rangle$ pure tilt grain boundaries (GB), has allowed the determination of atomic structures which are often different from the one originally proposed by Hornstra [5] or by Möller [6]. The only exceptions are the particularly simple case (111) $\Sigma=3$ and $(1 \overline{2} 2) \Sigma=9$ for which the predicted structures were confirmed [4]. This paper reviews the results obtained for more complicated GBs and summarizes several papers already published by the Grenoble groups working in the framework of the «ARC silicium polycristallin ».

Apart from the exact coincidence twins it is also interesting to study what happens for incommensurate GB interfaces. Small departure either from the exact coincidence angle or from the exact symmetry interface will introduce defects such as grain boundary dislocations (GBDs) or steps. The knowledge of the defect configuration is thus one of the important key to build more general GB structures. These defects were thought to be responsible for the electrical activity of GBs and it is important to know if this idea can be supported by the atomic arrangement.

\section{Experimental procedure.}

Germanium bulk bicrystals were grown using the Czochralski method under hydrogen atmosphere. The starting material is undoped germanium $(50 \Omega \mathrm{cm})$. Two initial seeds are carefully machined and positionned using $\mathrm{X}$-rays so that the orientation is as close as possible to the ideal coincidence (accuracy : few $0.01^{\circ}$ ). The bicrystal growth is not always successful : in particular very low index and


not produced by this method, but from a naturally twin crystal used as a seed. Intermediate $\Sigma$-values such as $(2 \overline{3} 3) \Sigma=11$ are easily obtained by this method. In the case of a high energy GB as (211) $\Sigma=3$ instabilities tend to appear : the GB is 
then only stable for [011] crystal growth and the GB is not able to expand laterally without splitting into several others GB. The reaction :

$$
\begin{aligned}
\{211\} \Sigma=3 \rightarrow\{111\} \Sigma=3 & +\{111\} \Sigma=3 \\
& +\{255\} \Sigma=27
\end{aligned}
$$

is commonly observed on one side. The $\{255\}$ $\Sigma=27$ portion was extracted from such a multiply twin crystal and studied. Similarly $\{144\} \Sigma=33$ has been extracted from the splitting of a $\{233\}$ $\Sigma=11$. The GBs geometrical characteristics are given in table $\mathrm{I}$.

Table I. - Geometrical characteristics of the [011] pure tilt GBs in diamond cubic structures.

\begin{tabular}{l|c|c|r|r}
$\Sigma$ value & $\begin{array}{c}\text { Rotation } \\
\text { angle (o) }\end{array}$ & $\begin{array}{c}\text { Grain boundary } \\
\text { plane }\end{array}$ & $\begin{array}{r}\text { Coincidence lattice } \\
\text { unit cell in (011) }\end{array}$ & \multicolumn{1}{|c}{$\begin{array}{c}\text { DSC vectors } \\
\text { in }(011)\end{array}$} \\
\hline$\Sigma=33$ & 18.94 & $(1 \overline{4} 4)$ & {$[1 \overline{4} 4] ; 1 / 2[81 \overline{1}]$} & $1 / 33[1 \overline{4} 4] ; 1 / 66[81 \overline{1}]$ \\
$\Sigma=27$ & 31.59 & $(2 \overline{5} 5)$ & $1 / 2[2 \overline{5} 5] ; \quad[51 \overline{1}]$ & $1 / 54[2 \overline{5} 5] ; 1 / 27[51 \overline{1}]$ \\
$\Sigma=9$ & 38.94 & $(1 \overline{2} 2)$ & {$[1 \overline{2} 2] ; 1 / 2[41 \overline{1}]$} & $1 / 9[1 \overline{2} 2] ; 1 / 18[41 \overline{1}]$ \\
$\Sigma=11$ & 50.53 & $(2 \overline{3} 3)$ & $1 / 2[2 \overline{3} 3] ; \quad[31 \overline{1}]$ & $1 / 22[2 \overline{3} 3] ; 1 / 11[31 \overline{1}]$ \\
$\Sigma=3$ & 70.53 & $(1 \overline{1} 1)$ & {$[1 \overline{1} 1] ; 1 / 2[21 \overline{1}]$} & $1 / 3[1 \overline{1} 1] ; 1 / 6[21 \overline{1}]$ \\
$\Sigma=3$ & 109.47 & $(2 \overline{1} 1)$ & {$[1 \overline{1} 1] ; 1 / 2[21 \overline{1}]$} & $1 / 3[1 \overline{1} 1] ; 1 / 6[21 \overline{1}]$
\end{tabular}

Thin slices are cut from the bicrystals (or multiply twin crystals) in the appropriate orientation, thinned by ion milling and observed by HREM at $200 \mathrm{keV}$. Two classes of complementary experiments can then be performed :

i) High energy electron diffraction (HEED).

ii) Imaging mode : conventional or HREM mode.

By combining HEED and HREM, the 2D symmetry group can then be determined unambiguously.

Computer simulations are often necessary in order to deduce the atomic structures of the GBs : with the present resolution limit of the order of $0.22 \mathrm{~nm}$ the diamond structure appears along [011] as a fcc lattice. Therefore the atomic model should be build using careful comparison between experimental and simulated images.

\section{Study of $(2 \overline{5} 5) \Sigma=27$.}

The HEED data from ( $2 \overline{5} 5) \Sigma=27$ is very difficult to interpret : this is due to the large unit cell of the 2D relaxations (see below) giving a large number of diffraction spots of very low intensity and moreover to the high defect content of this GB. Thus, the only available information comes from HREM images projected along [011]. The observed periodicity in the [51]] direction is the one given by the coincidence lattice (CL) i.e. $2.83 \mathrm{~nm}$ (Fig. 1a). This result was already obtained in silicon [7] showing once more that defect structures are isomorphous in $\mathrm{Si}$ and Ge. The GB can be described as successive facets along $\{110\}$ planes with a width successively close to $4 d_{0}$ and $3 a_{0}\left(d_{0}=1 / 4\langle 211\rangle a_{0}\right.$ and $a_{0}$ lattice parameter). These facets are organized on both a)




along the [011] common axis in Ge-200 kV; bright tunnels. The 7 atom-rings of the structure are visible as

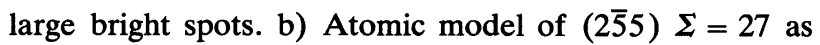
deduced from HREM. This model is identical to the one proposed by Vaudin et al. [7] and contains Lomer (L) units (7 and 5-atom rings).

halves so that a $\{110\}_{\mathrm{I}}$ facet is facing a $\{111\}_{\mathrm{II}}$ facet [8].

The atomic model proposed by Vaudin et al. [7] gives a nearly correct representation of the HREM images. This model (Fig. 1b) contains successive elements of $(1 \overline{2} 2) \Sigma=9 \mathrm{~GB}$, or L-unit with 5 and 7 atom rings, separated by one perfect chair shaped six membered ring symbolized hereafter by $u$. The sequence is :

$\mathrm{LL}^{\prime} \mathrm{u}\left(\mathrm{LL}^{\prime}\right)^{\prime}$ where the symbol ' denotes a mirror glide symmetry operation. The 7 atoms rings are particularly well visible when tunnels in the structure are white on HREM images. Their location corresponds to the atomic model and they are approximately situated at mirror glide symmetry related sites.

Indeed this model implies a complete mirror glide 
symmetry and there are no exact atomic coincidence sites. The associated rigid body translation (RBT) should then be equal to $t=1 / 54$ [511] ]. It has not yet been possible to verify this conclusion : the RBT should be measured on $(51 \overline{1})$ planes for which the lattice spacing is small. $t$ is equal to $0.027 \mathrm{~nm}$ and very close to the accuracy limit of HREM images. On the other hand the $\alpha$-fringes method is difficult to interpret because of the large defect density.

A large density of linear defects of the order of 0.5 to $1 \times 10^{7} \mathrm{~m}^{-1}$ is present along the [011] axis in the GB plane. Two types are found i) pure coherent steps $(30 \%)$ and ii) steps associated with dislocation $(70 \%)$.

The pure coherent steps as well as steps associated with GBD have a very well defined structure (Fig. 2). They are composed of a small microcrystal in twin orientation with one of the two crystals. Thus the $(2 \overline{5} 5) \Sigma=27$ is split following the reaction :

$$
\begin{aligned}
& (2 \overline{5} 5)_{\mathrm{I}} \Sigma=27 \rightarrow(11 \overline{1})_{\text {II }} \Sigma=3 \\
& +(12 \overline{2})_{\mathrm{I}} \Sigma=9 .
\end{aligned}
$$

The length of the splitting is proportional to the step height. At the end of the microcrystal a small surface of incoherent $(1 \overline{1} 1)_{\text {I }} \Sigma=9$ is formed. An atomic model of the reaction (2) is easy to build with progressive adjonction of T-units (boat shaped six atoms ring). A complete atomic structure of a coherent step is then available from HREM results (Fig. 2b) : its important feature is the appearance of difficult reconstruction scheme with a shear along


Fig. 2. - a) Coherent step in (255) $\Sigma=27$. This step is in fact formed by a small microcrystal limited by a $(11 \overline{1})$ $\Sigma=3$ and a $(12 \overline{2}) \Sigma=9$ and a very small portion of (1111) $\Sigma=9$. Ge-200 kV. Bright tunnels. b) Atomic model of the smallest coherent step in $(2 \overline{5} 5) \Sigma=27$. Note the two reconstructions $D_{1}$ and $D_{1}+S$.
[011] and symbolized by $D_{1}+S$ (double periodicity plus shear).

The GBDs are all of the same type and of the same sign : pure edge and increasing the misorientation angle. Therefore they are secondary dislocations with $b=1 / 54[2 \overline{5} 5]$ and the atomic model is very simple. A small portion visible on HREM picture consisting of 3 consecutive Lomer type structural units, introduces a typical $\Sigma=9$ configuration. The surprising fact that GBDs are nearly always associated with a large step (and a microcrystal) shows that there is an attraction between GBDs and steps.

\section{Study of $(2 \overline{3} 3) \Sigma=11$.}

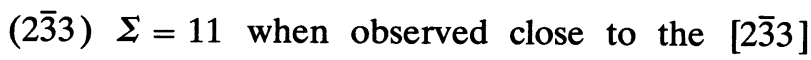
normal incidence gives clear diffraction pattern [9]. Extra matrix spots are identified in the $2 \overline{3} 3$ pole giving an apparent cell 1/2 [011]; [311] ]. However out of the $2 \overline{3} 3$ pole the fine GB spots are twice as numerous and give a true periodicity $1 / 2$ [011]; $2[31 \overline{1}]$. The period is thus twice the CL periodicity and is reduced by half when projected on the GB plane. As a consequence the $\Sigma=11$ bicrystal space group is $p m c^{\prime} 2{ }_{1}^{\prime}$ where $c^{\prime}$ is the $(2 \overline{3} 3)$ mirror glide plane. The HREM observations clearly confirm the previous result (Fig. 3). There is a total period along the GB plane equal to $3.75 \mathrm{~nm}$ (twice the CL) with a clear mirror glide symmetry operation. The RBT measurement using the $\alpha$-fringes method gives no


tion normal to the GB plane. Similarly HREM images give a dilatation equal to $\sim 0.04 \mathrm{~nm}$ and the $(31 \overline{1})$ planes are aligned within the accuracy limit $(0.025 \mathrm{~nm})$. As a conclusion the GB plane has a

a)



b)

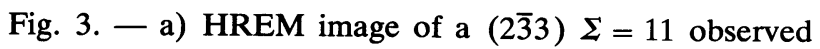
along the [011] common axis in Ge-200 kV. Bright tunnels. The 7 atom-rings of the structure are visible as large bright spots. The mirror glide symmetry operation is directly

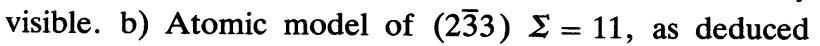
from HREM image. This model contains $L$ unit and Twin (T) unit, in addition with a new unit $t$ including 5 and 7 rings. 
$(2 \times 1)$ superstructure after relaxation similarly to what happens at clean $\{111\}$ surfaces.

Several models compatible with the observed [622] period were proposed by Papon et al. [9]. However they are not compatible with the HREM observations and a new model has been recently proposed [10]. In this model (Fig. 3b) the succession of structural units is the following :

\section{LtLTL' t' L'T}

where $t$ is a new unit including 5 and 7 atom rings. This succession incorporates not only elements from the (122) $\Sigma=9$ and from (1111) $\Sigma=3$ but also a new element $t$ which was proposed by Hornstra [5].

The density of linear defects in $(2 \overline{3} 3) \Sigma=11$ is relatively low $0.2-0.5 \times 10^{7} \mathrm{~m}^{-1}$. Usually they are not associated with steps although a single step is sometimes observed [10]. They all give a zero Burgers vector component on the (011) plane. However a large contrast variation of the atomic columns with a dark core region suggests the presence of a large shear strain parallel to the observations axis. Therefore these defects should be associated to screw 1/2 [011] dislocations : such GBDs give no step and no visible discontinuity when observed along [011].

The splitting of $(2 \overline{3} 3) \Sigma=11$ into two other GBs occurs frequently even at a macroscopic scale. The reaction is the following:

$$
\begin{aligned}
(2 \overline{3} 3)_{\mathrm{I}} \Sigma=11 \rightarrow(1 \overline{1} 1)_{\mathrm{I}} \Sigma= & 3 \\
& +(\overline{1} \overline{4} 4)_{\mathrm{II}} \Sigma=33 .
\end{aligned}
$$

\section{Study of $(21 \overline{1}) \Sigma=3$.}

On a $(21 \overline{1}) \Sigma=3$, HEED exhibits various superlattice spots. Firstly there are spots corresponding to the CL periodicity, but additional spots show that the GB plane has a $\mathrm{cm}$ planar symmetry. The unit cell is twice as large as the CL in two directions. The period is [011] $\times 2$ [1111].

The $\alpha$-fringes method on $\mathrm{Si}$ as well as on Ge gives a large RBT [11,12] along $\langle 111\rangle$ and a small dilatation component.

HREM confirms the $\mathrm{c}(2 \times 2)$ superstructure [13]. The apparent periodicity when observed along [011] axis is only $[\overline{1} 1 \overline{1}]$ (Fig. 4a). However when observed along the [231] axis a clear double periodicity is visible with a $0.74 \mathrm{~nm}$ repeat distance in accordance with the space group and the unit cell $[011] \times 2[\overline{1} 1 \overline{1}]$. The RBT is also measured to be : $\mathbf{t}=1 / 11[\overline{1} 1 \overline{1}]+1 / 20[21 \overline{1}]$.

Several atomic models of the (211) $\Sigma=3$ in semiconductors have been proposed : none of them
(1)



along the [011] common axis in Ge-200 keV. Bright atoms. Note the large rigid body translation (RBT) directly visible on 111 planes. b) Atomic model of the (211) $\Sigma=3$ as projected along the [011] common axis. Pair bonds are reconstructed at $R_{0}$ and $R_{1 / 2}$. c) Side view of the GB plane of the (211) $\Sigma=3$ showing the reconstruction scheme along 011 atomic rows.

are compatible with the $c(2 \times 2)$ superstructure. Papon and Petit [14] recently proposed a completely new one, compatible with both the symmetry and the HREM images. The structure (Fig. 4b) contains a very asymmetric arrangement with a pair of reconstructed bonds along the [011] common axis. The reconstruction, $\mathrm{D}_{1}$, doubles the periodicity along [011] and is similar to the one proposed for reconstructed $30^{\circ}$ partial dislocations [15]. The successive reconstructed rows are shifted by $1 / 2$ [011] forming the centred pattern and doubling the periodicity in two directions.

The successive structural units are then: (TT) associated with $L, R_{0}$, TT associated with $L$, $R_{1 / 2}$ where $R$ is a new unit containing five and eight atoms rings as seen from the [21ī] direction (Fig. 4c).

The density of linear defects in (21̄) $\Sigma=3$ is equal to 0.5 to $2 \times 10^{7} \mathrm{~m}^{-1}$. These defects are characterized by their variety. At least four different types can be distinguished [16]. i) Coherent steps not associated with GBD ; ii) $1 / 6\langle 211\rangle$ pure edge or $30^{\circ} \mathrm{GBD}$ with steps ; iii) $1 / 2\langle 110\rangle$ edge or $60^{\circ}$ including a change in RBT; iv) microcrystal formation.

\section{Study of $(1 \overline{1} 1)_{\text {II }} \mathbf{\Sigma}=9$.}

The incoherent $(1 \overline{1} 1)_{\text {II }} \Sigma=9$ has a very complex nature (Fig. 5a). There is a stair-case shape with successive microfacets along the 111 planes of either crystal I or II, thus creating a periodic triangular shape microcrystals. These microcrystals have three different facets : two $\{111\} \Sigma=3$ and one $\{522\}$ 



Fig. 5. - a) HREM of an incoherent (1111) $\Sigma=9$. Note the stairs formed by successive microcrystals. b) Atomic model of a). Note the large number of reconstruction along [011] in the $\{522\} \Sigma=3$ portion.

$\Sigma=3$. The periodicity of such an arrangement is equal to $1 / 2[25 \overline{5}]$ as given by the $\Sigma=9$ coincidence lattice. The atomic structure of such an incoherent GB incorporates already known elements as the Tunits or L-units, and the $7^{+}-5$ unit (Fig. 5b). The result is a complex, although periodic, $3 \mathrm{D}$, arrangement giving a GB «thickness » of $1.3 \mathrm{~nm}$.

\section{Discussion and conclusion.}

The important points of the present experimental results concern : i) the variety of new structural units discovered in periodic GBs as well as in defects : however there are no dangling bonds in any case ; ii) the possibility for a high energy GB to be splitted into lower energy parts forming microcrystals ; iii) the defect content which increases with GB energy.

New structural units, results from new reconstruction scheme. Up to now only reconstruction in the (011) plane was considered in pure tilt [011] GB. For symmetrical GBs and misorientation angle, $\theta$, up to $70.53^{\circ}$ this was enough to explain any GB structure by mixing three particular units [7]:

- the perfect chair-shaped 6 atom ring,

- the 5 and 7 atom rings. (L-unit) characteristic of a Lomer dislocation,

- the boat-shaped 6 atom-ring (T-unit).

Although exact, this mixing rule of simple structural units, is complicated by the fact that the exact distribution cannot be predicted from purely geometrical models. There is, in general, a tendency for introducing a mirror glide symmetry explaining that superstructure may well be favored (see for instance $\Sigma=11$ ). For larger misorientation angle or for asymmetrical GB reconstruction along the [011] common axis should be considered. A priori four reconstruction schemes could occur as described in [10]. Secondary dislocations introduced by small b) deviation from a high coincidence GB were proposed to be formed by incorporation of structural units of the closest high coincidence position [17]. This is true for small angle GB as $\Sigma=27$. However for larger angle this simple idea is not necessarily valid. For instance it was shown, that a $1 / 3$ [1111] GBD in (1111) $\Sigma=3$ which decreases $\theta$, does not incorporate elements of the closest twin i.e. (2533) $\Sigma=11$. Similarly the model presented for a $1 / 6$ [21ī] GBD in (2ī1) $\Sigma=3$, which increases $\theta$, does not incorporate elements of the (4111) $\Sigma=9$. For this later twin, with $\theta=141.06^{\circ}$, a model is proposed by Papon and Petit [14] and contains a $D_{2}$ reconstruction, not encountered in the $1 / 6$ [211] GBD model.

The splitting of high energy grain boundary into parts of lower energy is rather general. For instance the reaction

$$
(1 \overline{2} 2)_{\mathrm{I}} \Sigma=9 \rightarrow(1 \overline{1} 1)_{\mathrm{I}} \Sigma=3+(\overline{1} \overline{1} 1)_{\mathrm{II}} \Sigma=3
$$

is commonly observed at a macroscopic scale during crystal growth as well as several other reactions reported in this paper. These reactions may help in classifying the GB energies. For instance if one assumes that a splitting could only occur if it is energetically favorable, the series of reactions (1) to (4) lead to the conclusion :

$$
\begin{aligned}
\gamma(1 \overline{1} 1) \Sigma=3 & <\gamma(1 \overline{2} 2) \Sigma=9<\cdots \\
& \cdots<\gamma(2 \overline{5} 5) \Sigma=27<\gamma(21 \overline{1}) \Sigma=3
\end{aligned}
$$

where $\gamma$ is the GB energy. More systematic use of splitting reaction for energy determination is now under progress. It should be noted that, for high coincidence twin positions, the usual equilibrium equation at a triple point, for measuring surface energy, is completely inadequate. The reason is simply that the surface energy is very dependent of the GB plane in special symmetry positions. It has also been shown that microcrystal formation induced by microsplitting is an efficient way to built asymmetrical GB. It has been observed at (1111) steps in (255) $\Sigma=27$ as well as in a periodic manner at (111) $\Sigma=9$. Therefore it seems as if the GB «thickness" increases with the GB energy. It is limited to an atomic layer for coherent $\Sigma=3$ or


$\Sigma=3$ (2 atomic layers), and even larger for (1111) $\Sigma=27$ or (1111) $\Sigma=9$ (3 or 4 atomic layers).

The defect content of as grown GBs is very sensitive to their energy (or their complexity). An as-grown (1111) $\Sigma=3$ does not contain defects. A (122) $\Sigma=9$ has only very few defects in form of 
pure steps but no GBD. In (2555) $\Sigma=27$, the defect density increases but they are mainly associated to pure steps : in this case GBDs can be explained in terms of secondary dislocations or additional misorientation. Finally in (21̄) $\Sigma=3$ not only a large density of steps is present but also GBDs (not due to a misorientation). Qualitatively this correlation may be due to the fact that the GBD core energy is small when the atomic structure of the GB is largely distorted. Concerning the steps, the density variation between different GBs can also be explained with a similar argument : the surface energy of a complex GB is only weakly affected by small change in the GB plane.

It is worthwhile to note in conclusion that in the structures studied so far, it is always possible to build completely reconstructed models. Tetracoordination can be reestablished everywhere : in these conditions it is likely that no deep levels are introduced in the gap [18]. The electrical activity of some grain boundaries observed in as-grown case, as well as after annealing treatment should therefore be attributed to impurities or intrinsic point defects. This hypothesis, strongly supported by the present observation of $\langle 011\rangle \mathrm{GBs}$, is very similar to what has been recognized several years ago for dislocation cores. The electrical activity of dislocations is commonly attributed to intrinsic « point-defects » (kinks, solitons, vacancies, ...) or to segregated impurities (for a review see [19]). This idea could thus be generalized for $\langle 011\rangle$ GBs.

\section{References}

[1] For a review see: Polycrystalline semiconductors. Physical Properties and Applications, Ed. G. Harbeke (Springer Verlag, Berlin).

[2] Pond, R. C. and Vlachavas, D., Proc. R. Soc. A 386 (1983) 95.

[3] For a review see : Polycrystalline semiconductors. $J$. Physique Colloq. 43 (1982) C1.

[4] D'Anterroches, C. and Bourret, A., Philos. Mag.A 49 (1984) 783.

[5] Hornstra, J. R., Physica 25 (1959) 409.

[6] Moller, H. J., Philos. Mag. A 43 (1981) 1045.

[7] Vaudin, M. D., Cunningham, B. and Ast, D. G., Scripta Met. 17 (1983) 191.

[8] Bourret, A. and Bacmann, J. J., Surf. Sci. 162 (1985) 495.

[9] Papon, A. M., Petit, M. and Bacmann, J. J., Philos. Mag. A 49 (1984) 573.

[10] Bourret, A., Bacmann, J. J., Grain boundary structure and related phenomena. Proc. of JIMIS-4 Suppl. to Trans. Jpn. Inst. Met. (1986) 125.
[11] Vlachavas, D. and Pond, R. C., Inst. Phys. Conf. Ser. 60 (1981) 159.

[12] Rocher, A., Labidi, M., Revue Phys. Appl. 21 (1986) 201.

[13] Bourret, A., Billard, L. and Petit, M., Inst. Phys. Conf. Ser. 76 (1985) 23.

[14] Papon, A. M. and Petit, M., Scripta Met. 19 (1985) 391.

[15] Marklund, S., Phys. Status Solidi (b) 92 (1979) 83.

[16] Bourret, A. and BACMANN, J. J., Newcastle, EMAG Conf., Inst. Phys. Conf. Ser. 78 (1985) 337 (London, Adam Hilger Ltd).

[17] Sutton, A. P. and Vitek, V., Philos. Trans. R. Soc. Lond. A 309 (1983) 1.

[18] Mauger, A., Bourgouin, J. C. Allan, G., LANNOO, M., BourReT, A. and Billard, L., Phys. Rev. B 35 (1987) 1267.

[19] For a review see : Propriétés et structure des dislocations dans les semiconducteurs. J. Physique Colloq. (1983) C4-9. 